\title{
Accuracy of the tuberculin skin test for diagnosis of latent tuberculosis in population with high coverage of Bacillus Calmette-Guérin vaccination
}

\author{
Renata Báez-Saldaña1,2,3*, Lourdes García-García³, Leticia Ferreyra-Reryes ${ }^{3}$, Pablo Cruz-Hervert ${ }^{3}$, \\ Norma Mongua-Rodríguez ${ }^{3}$, Guadalupe Delgado-Sánchez ${ }^{3}$, Elizabeth Ferreira-Guerrero ${ }^{3}$, and \\ Adrián Rendón ${ }^{4}$ \\ ${ }^{1}$ Clinical Service of Oncological Pneumology, Instituto Nacional de Enfermedades Respiratorias, Mexico City; ${ }^{2}$ Subdivision of Medical Specializations, \\ Postgrade Division, School of Medicine, Universidad Nacional Autónoma de México, Mexico City; ${ }^{3}$ Infectious Disease Research Center, Instituto \\ Nacional de Salud Pública, Cuernavaca, Morelos; ${ }^{4}$ Center for Research, Prevention and Treatment of Respiratory Infections, Hospital Universitario \\ de Monterrey, Nuevo Leon. Mexico
}

\begin{abstract}
Introduction: The Mantoux tuberculin skin test (TST) for the diagnosis of latent tuberculosis infection (LTBI) has a variable performance and limited usefulness in Bacillus Calmette-Guérin (BCG)-vaccinated populations. Objective: The objective of the study was to evaluate the performance of the TST for the diagnosis of LTBI in household contacts of patients with active pulmonary tuberculosis (PTB). Materials and methods: The TST was administered to 113 cases with PTB and 194 household contacts. The gold standard was the bacteriological confirmation in patients. The household contacts were classified as true positives according to the selected cutoff point. Those with purified protein derivative reactivity less than the cutoff point but different from zero were classified as false positives. The area under the curve was measured and the best cutoff point was determined. Sensitivity, specificity, positive and negative predictive values (PV), and positive and negative likelihood ratios $(L R)$ were measured with $2 \times 2$ contingency tables. Results: The best cutoff point was at $10 \mathrm{~mm}$ of induration, with an area under the curve of 0.93 , sensitivity $85 \%$, specificity $88 \%$, positive PV $94 \%$, negative PV $71 \%$, positive LR 6.9, and negative $L R$ 0.17. There was no association between reactivity to TST and BCG vaccination, OR (95\% confidence interval Cl) 1.14 (0.65-2.10), $p=0.644$. Conclusions: The TST showed a very good performance, as it lead to an important change from pre-test to post-test probability.
\end{abstract}

Key words: Latent tuberculosis. Tuberculin skin test. Specificity. Bacillus Calmette-Guérin vaccination.

\section{Introduction}

Although tuberculosis (TB) is a preventable, diagnosable, and curable disease, it killed 1.3 million people worldwide in $2017^{1}$. Many are the challenges that must be faced for its control and eradication, due to the biological complexity of the disease. The priority for TB control is the diagnosis and treatment of infectious cases and those with the active disease. However, the identification and treatment of subjects with latent TB at high risk of developing the active disease are a critical component for its eradication. The study and diagnosis of latent TB infection (LTBI) have a place of utmost importance in the dynamics of TB epidemiology in the community. There are 2 billion with latent infection in the world, which represent the disease reservoir, as $10 \%$ of them will develop the disease at some point in their lives, referring to immunocompetent subjects ${ }^{2,3}$.

For over a century, the tuberculin skin test (TST) has been the only tool for diagnosing LTBI. At present, in
Corrrespondence:

*Renata Báez-Saldaña

E-mail: baezrd@unam.mx
Available online: $21-07-2020$ Rev Med Hosp Gen Mex. 2020;83(3):120-126 www.hospitalgeneral.mx 0185-1063/@ 2020 Sociedad Médica del Hospital General de Mexico. Published by Permanyer. This is an open access article under the CC BYNC-ND license (http://creativecommons.org/licenses/by-nc-nd/4.0/). 
addition to this method, there is an in vitro test based on the production of interferon gamma by peripheral T-lymphocytes (Interferon gamma release assay IGRA). Both tests perform well and are deemed adequate for the diagnosis of LTBI, although IGRA has more specificity than the TST in a Bacillus CalmetteGuérin (BCG)-vaccinated population, particularly if they were vaccinated after childhood. The World Health Organization algorithm for the study and treatment of LTBI strongly recommends that both TST and IGRA can be used to diagnose $\mathrm{LTBI}^{2}$.

TST specificity is variable as it can be modified by the presence of environmental mycobacteria and BCG vaccination history in the population, due to the fact that both situations lead to purified protein derivative (PPD) cross-reactivity. Hence, it is proposed that studies should be carried out in each region or community to identify the best cutoff point. Thenceforth, its performance as a diagnostic test should be evaluated for its correct interpretation, for the identification of subjects with LTBI at high risk of developing active TB, which could benefit from preventive treatment and thereby interrupt the endemic transmission chain ${ }^{4}$.

Positive and negative predictive values (PV), as well as their specificity at different cutoff points, are unknown in the Mexican population, whose BCG vaccination coverage is high. The objective of this study was to identify, by means of the PPD tuberculin test, the best cutoff point and its performance to differentiate LTBI, in household contacts of patients with active pulmonary TB (PTB). In addition, the relationship between TST response and $B C G$ vaccination history, body mass index, and the duration of the disease (the latter only in patients) was evaluated.

\section{Materials and methods}

The institutional ethics committee approved the study. Its design was prospective cross-sectional, in patients with bacteriologically proven PTB and their household contacts at the outpatient and hospitalization service of a third-level health-care institution, which is a national health-care center of reference. The inclusion criteria for patients were cases of patients aged 15 years or older with bacteriologically proven PTB, by either smear microscopy or positive sputum culture. Those patients undergoing immunosuppressive treatment, with diabetes or human immunodeficiency virus (HIV) infection were excluded in the study. The inclusion criteria for the household contacts were of any age, who stated that they lived in the patient's home from the onset of the patient's symptoms and with a chest X-ray with no evidence of pleural or lung lesions. Exclusion criteria were the same as for the patients. Regarding the patients, universal variables, habits, and $B C G$ vaccination history that was verified by the presence of scar and duration of the disease were studied. In the case of BCG vaccination history, the patient or responsible family member was asked about this aspect; if the answer was affirmative, the presence of a scar was sought and if it was present, subjects were considered as vaccinated. If the answer was "no," the presence of a scar was also sought, and if not found, subjects were classified as unvaccinated. There were very few cases (less than about $2 \%$ ), who mentioned having a vaccination history that was not corroborated by scarring and vice versa. No records were collected from this minority. Regarding the measurement of the disease duration, the time was measured in days in which the patient started with respiratory symptoms until the moment of the diagnosis of PTB. In those patients with risk factors for HIV infection, the ELISA test was requested.

All the patients previously had a clinical history, chest $X$-ray, and positive sputum smear and were under anti-TB therapy. A complete medical history was made about the household contacts, universal variables, habits, and BCG vaccination history were studied, which was verified by the presence of a scar and chest X-ray. In those household contacts, who reported coughing and expectoration, a sputum smear was requested to rule out the active form of the disease.

In patients as in household contacts, PPD-S (Tubersol ${ }^{\circledR}$ ) was applied using the Mantoux technique following international recommendations ${ }^{5,6}$.

All the household contacts that presented abnormal chest X-rays, and/or positive sputum smear were referred and studied, as well as those who met criteria for treatment of latent TB, according to the criteria established by the Official Mexican Standards for these cases.

\section{Statistical analysis}

The statistical package Stata ${ }^{\circledR}$ v15.1 was used. To summarize the data, the appropriate descriptive statistics were performed for each variable. For the numerical variables, the median and interquartile range (25-75) were used, and for the categorical variables, frequency, and percentage.

The response of the PPD tuberculin test was graphed, according to the variation of the level of its positivity by 


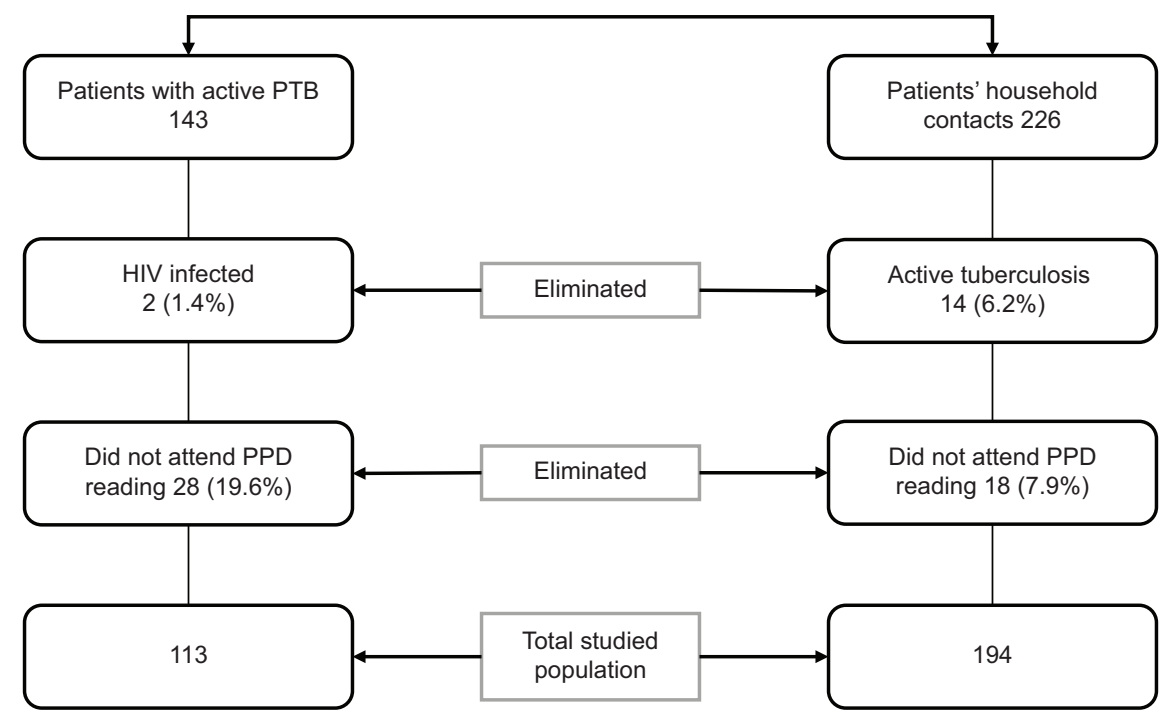

Figure 1. Flow chart of patients with active tuberculosis and household contacts.

means of the receiver operating characteristics (ROC) curve, with which the area under the curve was measured and the best cutoff point was identified. The estimation of the TST sensitivity was based on the TST reaction in patients with PTB in whom the gold standard was the bacteriological verification of TB by smear microscopy and/or sputum culture. The specificity estimate was based on the contact reactions of patients with the active form of the disease.

To measure the test's performance, a $2 \times 2$ contingency table was used and the sensitivity, specificity, positive and negative $\mathrm{PV}$, and likelihood ratios at cutoff points of 5,10 , and $15 \mathrm{~mm}$ induration were measured. Household contacts were classified as true positives from the selected cutoff point, and those household contacts with PPD-S reactivity less than the cutoff point but different from zero were classified as false positives.

The association between reactivity to the TST as a dichotomous variable (according to the selected cutoff point) and BCG vaccination history, body mass index $>17$, and the duration of the disease was evaluated using bivariate logistic regression analysis (the latter only in patients). The association between active PTB and $B C G$ vaccine was also measured.

\section{Results}

One hundred and forty-three patients and 226 household contacts were initially recruited. From this total, 30 patients $(21 \%)$ were eliminated, because 28 of them did not attend the PPD reading and two were infected with HIV. Regarding the household contacts studied, 14 cases $(6.2 \%)$ with active PTB were documented, so they were included in the patients' group and 18 household contacts $(7.9 \%)$ were eliminated as they did not attend the PPD reading. In total, 113 patients and 194 household contacts of the same patients were studied (Fig. 1).

As for the patients with active PTB, $74(65.49 \%)$ were new cases, 10 (8.85\%) fail or relapse, and 29 (25.66\%) were multidrug-resistant cases. The median (interquartile range) of the induration diameter in millimeters for each group was 13 (1-17), 11 (10-17), and 15 (11-18), respectively, without statistically significant differences $(p=0.4357)$.

The patients were older than the household contacts, predominantly consisting of males and smokers. Likewise, the frequency of BCG vaccination history and body mass index was lower than in the group of household contacts (Table 1).

The distribution of reactivity frequencies in patients with active PTB was bimodal. On the one hand, because of the non-reactivity frequency $(0 \mathrm{~mm}$ of induration) and the reactivity curve with a tendency to normality, whose reactivity frequency mode was at 10 and $20 \mathrm{~mm}$ induration equally. In the household contacts, the reactivity frequency curve showed a bias to the right, with a mode of $20 \mathrm{~mm}$ of induration (Table 2).

According to the results of the probability indices and the area under the curve, the best cutoff point in our population was between 10 and $11 \mathrm{~mm}$ of induration. The test's 
Table 1. Overall clinical characteristics of patients with active PTB and household contacts

\begin{tabular}{|l|c|c|c|}
\hline & Patients with PTB $\mathbf{n = 1 1 3}$ & Household contacts $\mathbf{n = 1 9 4}$ & $\mathbf{p}$ \\
\hline Age: years* & $39(26-50)(16-91)$ & $20.5(10-37)(1-81)$ & 0.0001 \\
\hline $\begin{array}{l}\text { Sex } \\
\text { Males } \\
\text { Females }\end{array}$ & $63(56 \%)$ & $85(44 \%)$ & 0.044 \\
\hline Smokers & $50(44 \%)$ & $109(56 \%)$ & 0.024 \\
\hline BCG vaccination history & $16(14 \%)$ & $12(6 \%)$ & 0.000 \\
\hline Body mass index* & $56 / 102(55 \%)$ & $157 / 175(90 \%)$ & 0.001 \\
\hline Duration of disease months* & $19(18-22)(12-33)$ & $22.5(18-26)(13-50)$ & \\
\hline
\end{tabular}

The data are in frequencies and percentage. ${ }^{*}$ Median (IOR) (minimum-maximum). BCG: Bacillus Calmette-Guérin; IQR: interquartile range; PTB: pulmonary tuberculosis.

Table 2. Reactivity of the PPD-S tuberculin test in patients with active pulmonary tuberculosis and household contacts

\begin{tabular}{|l|c|c|}
\hline & Patients $\mathbf{n = 1 1 3}$ & $\begin{array}{c}\text { Household } \\
\text { contacts } \mathbf{n = 1 9 4}\end{array}$ \\
\hline Mean (SD) & $12(8) \mathrm{mm}$ & $10(11) \mathrm{mm}$ \\
\hline Median (IQR) & $14(7-17) \mathrm{mm}$ & $10(0-18) \mathrm{mm}$ \\
\hline Mode & $10 \mathrm{and} 20 \mathrm{~mm}$ & $20 \mathrm{~mm}$ \\
\hline Maximum/minimum value & $0-30 \mathrm{~mm}$ & $0-68 \mathrm{~mm}$ \\
\hline 0 mm induration & $28(24.78 \%)$ & $78(40.21 \%)$ \\
\hline $3-9 \mathrm{~mm}$ & $4(3.53 \%)$ & $11(5.67 \%)$ \\
\hline$>5 \mathrm{~mm}$ & $85(75.22 \%)$ & $115(59.27 \%)$ \\
\hline$>10 \mathrm{~mm}$ & $81(71.68 \%)$ & $105(54.12 \%)$ \\
\hline$>15 \mathrm{~mm}$ & $54(47.79 \%)$ & $76(39.17 \%)$ \\
\hline
\end{tabular}

IQR: interquartile range; SD: standard deviation.

performance after $10 \mathrm{~mm}$ of induration showed sensitivity, specificity, and positive PV above $85 \%$, with a negative PV of $70.91 \%$, and with positive and negative probability indices of 6.19 and 0.17 , respectively (Table 3).

The prevalence of LTBI was similar at 5 and $10 \mathrm{~mm}$ induration, 72.9 and $71 \%$ respectively, and approximately 10 points less with induration at $15 \mathrm{~mm}$. The area under the curve at the $10 \mathrm{~mm}$ cutoff point was 0.93 (Fig. 2).

The BCG vaccination history, the duration of the disease of the patients with active disease (as an approximation of the exposure time of the household contacts), as well as the body mass index in patients as in household contacts, did not present an association with the level of reactivity to PPD-S > $10 \mathrm{~mm}$ induration (Table 4).
The association between active PTB and BCG vaccination history proved to be protective OR $(95 \%$ confidence interval $[\mathrm{Cl}]) 0.14(0.07-0.26)$.

\section{Discussion}

This study provides valid information for the Mexican population (characterized by high coverage of BCG vaccination at birth), on the performance of TST for the diagnosis of LTBI in a high-risk group for developing TB disease, such as household contacts of patients with active TB. LTBI is defined as a state of persistent immune response to previously acquired Mycobacterium tuberculosis (MTB) antigens, which is not accompanied by clinical manifestations of active TB, so the infected subject presents no signs or symptoms, nor radiographic or bacterial evidence of disease. Whether by means of smear microscopy or sputum culture, subjects are not a source of transmission. We documented that the best cutoff point to identify LTBI using TST in the household contacts of patients with active PTB was between 10 and $11 \mathrm{~mm}$ of induration, demonstrating good performance as a diagnostic test, taking into account the test's accuracy indicators.

TST specificity is not predictable for different regions of the world, due to the large variation in the prevalence of environmental mycobacteria that produce cross-reaction, as well as BCG vaccination, although the reactivity produced by both is known to be low in comparison with MTB infection. In our study, the intermediate values $(3-9 \mathrm{~mm})$ showed a very low frequency in patients and household contacts, $3 \%$ and $5 \%$, respectively, suggesting the little influence of BCG vaccination and/or environmental mycobacteria in our population, a statement that is strengthened by the absence of 
Table 3. Accuracy of the PPD tuberculin test for the diagnosis of latent tuberculosis at 5, 10, and $15 \mathrm{~mm}$ induration as cutoff points

\begin{tabular}{|c|c|c|c|}
\hline & \multicolumn{3}{|c|}{ Cutoff points } \\
\hline Diagnostic indicators & $5 \mathrm{~mm}$ & $10 \mathrm{~mm}$ & $15 \mathrm{~mm}$ \\
\hline $\begin{array}{c}\text { Sensitivity \% } \\
(\mathrm{Cl} 95 \%) \\
\mathrm{a} /(\mathrm{a}+\mathrm{c})\end{array}$ & $\begin{array}{c}87.50 \\
(83.80-91.20)\end{array}$ & $\begin{array}{c}85.32 \\
(81.36-89.28)\end{array}$ & $\begin{array}{c}68.78 \\
(63.60-73.97)\end{array}$ \\
\hline $\begin{array}{l}\text { Specificity \% } \\
\qquad(C I 95 \%) \\
d /(b+d)\end{array}$ & $\begin{array}{c}93.98 \\
(91.31-96.64)\end{array}$ & $\begin{array}{c}87.64 \% \\
(83.96 \% 91.32)\end{array}$ & $\begin{array}{c}66.95 \\
(61.69-72.21)\end{array}$ \\
\hline $\begin{array}{l}\text { VPP } \% \\
\qquad(\mathrm{CI} 95 \%) \\
\mathrm{a} /(\mathrm{a}+\mathrm{b})\end{array}$ & $\begin{array}{c}97.51 \\
(95.77-99.25)\end{array}$ & $\begin{array}{c}94.42 \\
(91.85-96.98)\end{array}$ & $\begin{array}{c}76.92 \\
(72.21-81.64)\end{array}$ \\
\hline $\begin{array}{l}\text { NPV } \% \\
(\text { CI } 95 \%) \\
d /(c+d)\end{array}$ & $\begin{array}{c}73.58 \\
(68.65-78.52)\end{array}$ & $\begin{array}{c}70.91 \\
(65.83-75.99)\end{array}$ & $\begin{array}{c}57.25 \\
(51.71-62.78)\end{array}$ \\
\hline Positive probability index sensitivity/(1 - specificity) & 3.59 & 6.9 & 2.08 \\
\hline Negative probability index (1 - sensitivity)/specificity & 0.03 & 0.17 & 0.47 \\
\hline Prevalence $(a+c) /(a+b+c+d)$ & $72.96(60-77.93)$ & $71.01(65.93-76.09)$ & $61.56(56.12-67)$ \\
\hline Area under the curve & $0.49(0.41-0.57)$ & $0.93(0.90-0.95)$ & $0.88(0.84-0.92)$ \\
\hline
\end{tabular}

a: true positives; b: false positives; $c$ : false negatives; Cl: confidence interval; d: true negatives; NPV: negative predictive value: PPD: Purified protein derivative; PPV: positive predictive value.

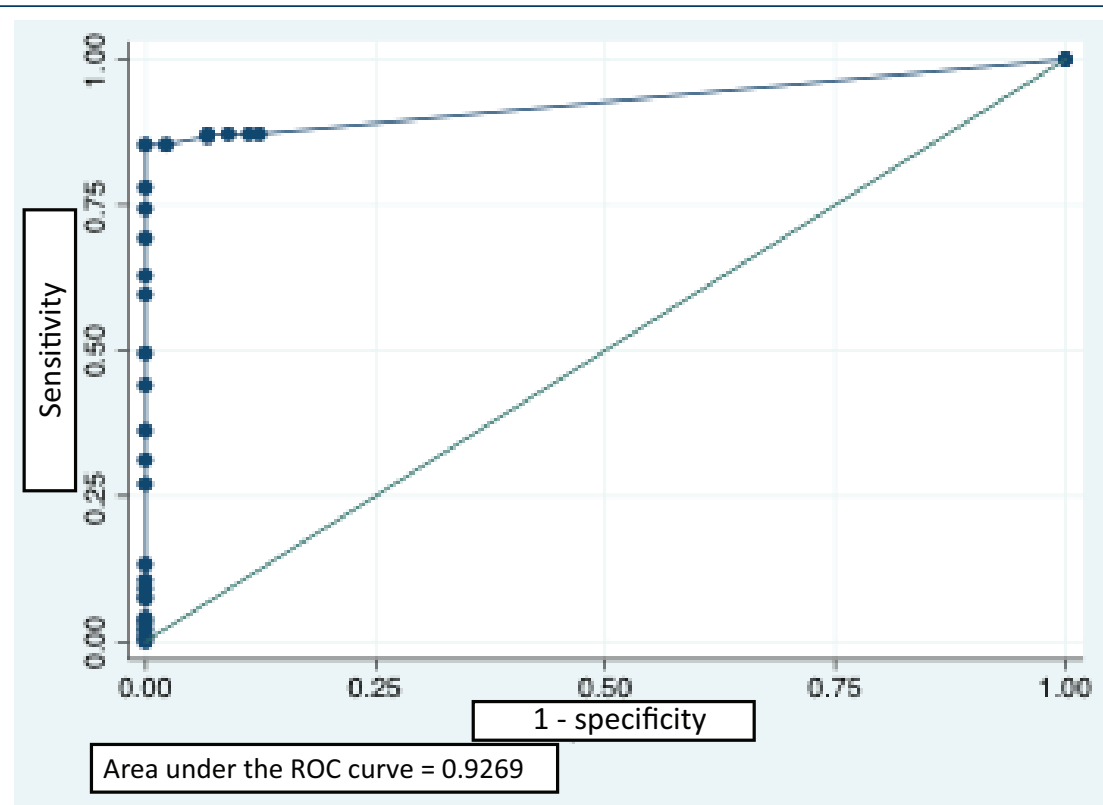

Figure 2. Receiver ROC curve of the tuberculin skin test PPD for latent tuberculosis diagnosis. PPD: Purified protein derivative; ROC: operating characteristic.

association between TST reactivity and BCG vaccination history in household contacts. This result is consistent with that of a study conducted in Mexican children aged 6-12 years, which demonstrated an independent association between being a close contact of a patient with active PTB and TST reactivity $(>10 \mathrm{~mm})$ 
Table 4. Association between reactivity to the tuberculin test at a cutoff point $>10 \mathrm{~mm}$ of induration and selected variables*

\begin{tabular}{|l|c|c|c|}
\hline & $\mathbf{n} / \mathbf{N}$ & $\mathbf{O R}(\mathbf{C l} 95 \%)$ & $\mathbf{p}$-value \\
\hline $\begin{array}{l}\text { BCG vaccination history in } \\
\text { the total population }\end{array}$ & $277 / 307$ & $1.14(0.65-2.01)$ & 0.644 \\
\hline $\begin{array}{l}\text { BCG vaccination history in } \\
\text { patients with TBP }\end{array}$ & $102 / 113$ & $2.35(0.99-5.63)$ & 0.054 \\
\hline $\begin{array}{l}\text { BCG vaccination history in } \\
\text { household contacts }\end{array}$ & $175 / 194$ & $1.21(0.46-3.21)$ & 0.700 \\
\hline $\begin{array}{l}\text { Body mass index }>17 \text { in the } \\
\text { total population }\end{array}$ & $275 / 307$ & $1.45(0.80-2.64)$ & 0.214 \\
\hline $\begin{array}{l}\text { Body mass index }>17 \text { in } \\
\text { patients }\end{array}$ & $105 / 113$ & $2.52(0.98-6.48)$ & 0.054 \\
\hline $\begin{array}{l}\text { Body mass index }>17 \text { in } \\
\text { household contacts }\end{array}$ & $170 / 194$ & $1.12(0.51-2.44)$ & 0.782 \\
\hline $\begin{array}{l}\text { Duration of tuberculosis } \\
\text { disease }\end{array}$ & $105 / 113$ & $1.00(0.99-1.01)$ & 0.153 \\
\hline
\end{tabular}

*Bivariate logistic regression. BCG: Bacillus Calmette-Guérin; Cl: confidence interval; OR: odds ratio.

(odds ratio $[\mathrm{OR}] 6.56,95 \% \mathrm{Cl} 2.05-21.17)^{7}$. However, it has been documented that the effect of BCG vaccination received in childhood against TST reactivity is minimal, especially 10 years after the application of the vaccine. This finding is present in $77.3 \%$ of household contacts, and although the remaining $22.7 \%$ are younger than 10 years, we consider that there is a great probability that a reactivity $>10 \mathrm{~mm}$ in the study population is the result of an infection rather than of a vaccine origin, because of the considerations previously described 8,9 .

The TST performance analysis based on the criteria of modern epidemiology, showed that for a contact of a patient with PTB, with TST $>10 \mathrm{~mm}$, the probability that the contact actually has LTBI after the test is $94.4 \%$. On the other hand, a negative result or PPD $<10 \mathrm{~mm}$ does not rule out the probability of LTBI in $29.1 \%$ of cases given the negative PV.

In this sense, the uncertainty regarding the LTBI has gone from the initial $71 \%$ or probability before the test (prevalence) toward probabilities of $94.4 \%$ or $29.1 \%$, this being a clinically important shift.

In this context, according to the positive probability index that resulted in our study, a patient's contact with bacteriologically proven PTB and PPD $>10 \mathrm{~mm}$, will be 6.9 times more likely to present latent TB, compared to a subject with a negative result or PPD $<10 \mathrm{~mm}$.
Approaches to measure the prevalence of LTBI taking into account the variables that cause cross-reaction such as BCG vaccination history and environmental mycobacteria show that at any chosen cutoff point, a proportion of the subjects subjected to the test will be poorly classified. In this study, the test's accuracy is greatly reduced in all its indicators anti-TB treatment when we move the intersection to indurations $>15 \mathrm{~mm}$. In contrast, indurations of 5 and $10 \mathrm{~mm}$, presented much better performance with similar accuracy for both cutoff points (Table 3). Measuring the prevalence of LTBI in a population is only possible if both sensitivity and specificity are known. The prevalence in the population of our study at 5 and $10 \mathrm{~mm}$ was very similar, $73 \%$ and $71 \%$, respectively, and approximately ten points less with induration at $15 \mathrm{~mm}$.

In a meta-analysis of 20 studies, $\mathrm{Pai}^{10}$ showed that TST sensitivity was heterogeneous, with a pooled estimate of $77 \%$ (71-82), the specificity in the population without BCG vaccination was $97 \%$ (95-99). However, in vaccinated subjects, this specificity was heterogeneous and low 59\% (46-73), and which according to the authors, show the main limitation of TST, because of the possibility of the aforementioned cross-reactions. In the case of our population, we can assume that the test was not affected by the BCG vaccination history.

Regarding the validity of the test, we will mention that in the case of patients, the comparison with the reference standard (sputum culture for MTB and/or bacilloscopy) was not independent or blind, because the inclusion criteria were that the patient had bacteriologically proven PTB. On the other hand, it is not possible to carry out this reference standard in household contacts, due to the LTBI's own biology. However, being a patient or contact has not influenced the result of the diagnostic test, because the TST application process was standardized.

The protection offered by the BCG vaccine for PTB in adults is variable. We evaluated this association, which was highly protective in our population with OR $(95 \% \mathrm{Cl}) 0.14(0.07-0.26)$ similar to a study carried out in the UK that showed an OR 0.22 (0.160.319). A meta-analysis study on the current evidence of protection provided by the BCG vaccine for TB shows that its efficacy decreases over time and is variable, with a faster decline in latitudes far from the equator and in people with mycobacterial sensitization assessed for TST before administration of the vaccine. The efficacy in children without prior 
sensitization to MTB or environmental mycobacteria shows an OR $(95 \% \mathrm{Cl}) 0.26(0.18-0.37)^{11,12}$. It is possible that the protection we observed in our study is related to our population being routinely vaccinated at birth. Therefore, we can assume that it is a population without prior sensitization to MTB or environmental mycobacteria.

Among the limitations of this study, it is necessary to consider that the sensitivity and specificity of the test for active TB do not translate into the accuracy for LTBI, which cannot be directly estimated. This is due to the lack of a gold standard in the household contacts, not because of a methodological deficiency of the study, but as previously commented, it is not possible to evaluate it, due to the LTBI biology. Likewise, the intra- and inter-observer variability of the TST measurement was not evaluated. Another limitation of the study is the absence of records from patients, who mentioned having a vaccination history that was not confirmed with scarring and vice versa. This is important, because of the BCG vaccinated versus unvaccinated categorization, which undoubtedly constitutes a source of error. However, these cases were very few, as mentioned in the material and methods part, they were approximately $<2 \%$ in our study sample. Thus, we consider that this aspect can have little effect on the validity of the results.

Several unanswered questions follow, one of which is whether the test has the ability to predict which individuals will progress to the active form of the disease and thus benefit from preventive therapy. Conversely, it is difficult to impose a cutoff point in the entire population as it is mandatory to assess the risk of disease in each group, and this will greatly depend on the prevalence of infection in each region, which can also vary over time.

\section{Conclusions}

TST $>10 \mathrm{~mm}$ at the study site is useful in our practice, because it leads to a significant change in the probability before the test against the probability after the test. The BCG vaccination history did not affect the performance of the test. Hence, it continues to be useful for the diagnosis of ITBL in the assessed population.

\section{Sources of funding}

The development of this study did not receive specific funding. The study was conducted with the support of Instituto Nacional de Enfermedades Respiratorias (National Institute of Respiratory Diseases).

\section{Conflicts of interest}

The authors have not conflicts of interest.

\section{Ethical disclosures}

Protection of human and animal subjects. The authors declare that no experiments were performed on humans or animals for this study.

Confidentiality of data. The authors declare that they have followed the protocols of their work center on the publication of patient data.

Right to privacy and informed consent. The authors declare that no patient data appear in this article.

\section{References}

1. World Health Organization. Global Tuberculosis Report; 2018. Available from: https://www.who.int/tb/publications/global_report/en. [Last accessed on 2019 Jun 07].

2. Guidelines on the Management of Latent Tuberculosis Infection. Geneva: World Health Organization. WHO/HTMTB/2015.01.

3. Cardona PJ, Ruiz-Manzano J. On the nature of Mycobacterium tuberculosis-latent bacilli. Eur Respir J. 2004;24:1044-51.

4. Rieder $\mathrm{HL}$. The dynamics of tuberculosis epidemiology. Indian J Tuberc. 2014;6:19-29.

5. Kiazyk S, Ball TB. Latent tuberculosis infection: an overview. Can Commun Dis Rep. 2017;43:62-6.

6. Centers for Diseases Control and Prevention. Latent TB Infection and TB Disease; 2016. Available from: https://www.cdc.gov/tb/topic/basics/tbinfectiondisease.htm. [Last accessed on 2019 Apr 07].

7. García-Sancho MC, García-García L, Jiménez-Corona ME, Palacios-Martínez M, Ferreyra-Reyes LD, Canizalez-Quintero S, et al. Is tuberculin skin testing useful to diagnose latent tuberculosis in BCG-vaccinated children? Int J Epidemiol. 2006;35:1447-54.

8. Farhat M, Greenaway C, Pai M, Menzies D. False-positive tuberculin skin tests: what is the absolute effect of BCG and non-tuberculous mycobacteria? Int J Tuberc Lung Dis. 2006;10:1192-204.

9. Bugiani M, Borraccino A, Migliore E, Carosso A, Piccioni P, Cavallero M, et al. Tuberculin reactivity in adult BCG-vaccinated subjects: a cross-sectional study. Int J Tuberc Lung Dis. 2003;7:320-6.

10. Pai M, Zwerling A, Menzies D. Systematic review: t-cell-based assays for the diagnosis of latent tuberculosis infection: an update. Ann Intern Med. 2008;149:177-84.

11. Mangtani P, Abubakar I, Ariti C, Beynon R, Pimpim L, Fine PE, et al. Protection by BCG vaccine against tuberculosis: a systematic review of randomized controlled trials. Clin Infect Dis. 2014;58:470-80.

12. Araujo $\mathrm{Z}$, de Waard $\mathrm{JH}$, de Larrea CF, Borges $\mathrm{R}$, Convit J. The effect of bacille calmette-guérin vaccine on tuberculin reactivity in indigenous children from communities with high prevalence of tuberculosis. Vaccine. 2008;26:5575-81. 